\title{
ALTERACIONES EN LA CRONOLOGÍA Y SECUENCIA DE ERUPCIÓN DE DIENTES PRIMARIOS ASOCIADAS A FACTORES QUE INFLUYERON HASTA LA VIGÉSIMA SEMANA DE GESTACIÓN DE PACIENTES QUE ACUDEN AL CENTRO DE SALUD MATERNO INFANTIL PACHACÚTEC PERÚ-COREA (CALLAO, 2017)
}

\author{
ALTERATIONS IN THE CHRONOLOGY AND SEQUENCE OF ERUPTION OF PRIMARY \\ TEETH ASSOCIATED WITH FACTORS THAT INFLUENCED UP TO THE TWENTY- \\ SECOND WEEK OF GESTATION OF PATIENTS WHO COME TO THE CENTER OF \\ MATERNAL CHILDHOOD HEALTH PACHACUTEC PERU-COREA (CALLAO, 2017)
}

\author{
Luciano Álvaro Ordóñez ${ }^{1}$ \\ lucianoalvaro@hotmail.com \\ ORCID: 0000-0003-2868-6924 \\ Denisse Pilar Carmen Aguilar Gálvez' \\ daguilar@cientifica.edu.pe \\ ORCID: 0000-0002-8394-7173
}

\section{RESUMEN}

Objetivo: Determinar la asociación entre los factores internos que influyen en la gestante hasta la vigésima semana de gestación y que producen alteraciones en la cronología y la secuencia de erupción de los dientes primarios de sus niños. Metodología: El presente estudio fue observacional, analítico y retroprospectivo. La población y muestra estuvo compuesta por 150 niños, atendidos en el servicio de crecimiento y desarrollo (CRED) que presentaron alguna alteración en la cronología o secuencia de erupción de los dientes temporal, y las historias clínicas de sus respectivas madres, que acuden al Centro de Salud Materno Infantil Pachacútec Perú-Corea (Callao, 2017). Para ello, debieron cumplir los siguientes criterios de inclusión: madres que acepten participar con su menor hijo en el proyecto de investigación, niños entre 6 y 31 meses que acuden al servicio de crecimiento y desarrollo (CRED), que presenten alguna alteración en la cronología o secuencia de erupción dental. Niños con peso y talla normal, y con lactancia exclusiva los primeros seis meses de vida. Madres que tengan sus historias clínicas completas con sus controles de gestación, que acudan al centro de salud y tengan entre 20 y 35 años. Los criterios de exclusión fueron los siguientes: madres o niños que no pertenezcan a la jurisdicción del centro de salud, niños con desnutrición o con alimentación basada en fórmula (artificial), niños con algún síndrome o prematuros, niños con enfermedades sistémicas. Resultados: Los factores que influyeron en la gestación

Citar como: Ordóñez L y Aquilar D. Alteraciones en la cronología y secuencia de erupción de dientes primarios asociadas a factores que influyeron hasta la vigésima semana de gestación de pacientes que acuden al Centro de Salud Materno Infantil Pachacútec Perú-Corea (Callao, 2017). Rev Cient Odontol (Lima). 2019; 7 (2): 11-22.

División de Odontopediatría, Carrera de Estomatología, Facultad de Ciencias de la Salud, Universidad Científica del SUR. LIMA, PeRÚ. 
se evaluaron por medio de la prueba estadística Chi cuadrado y se trabajaron a un nivel de significancia de 0,05. Al analizar la asociación entre las enfermedades infecciosas y las alteraciones en la cronología se encontró que en enfermedades como las ITU, que son las más comunes $(82,8 \%)$, presentó un nivel de significancia $p=0,000$. En el caso de la asociación entre las enfermedades infecciosas y las alteraciones en la secuencia se encontró que, en las enfermedades como las ITU, un $88.1 \%$ presentaron un nivel de significancia $p=$ 0,008 . Se hallaron porcentajes menores en enfermedades como la hipertensión $(4,1 \%)$, la salud mental $(3,3 \%)$ y enfermedades nutricionales $(9,8 \%)$, en lo que respecta a la cronología En el caso de la secuencia, fueron la hipertensión (2,4\%), la salud mental $(2,4 \%)$ y las enfermedades nutricionales $(7,1 \%)$. Con respecto al sexo y la asociación con alteraciones en la cronología de erupción, los resultados fueron del $49,2 \%$ para el sexo femenino y el $50,8 \%$ para el masculino $(p=0,271)$. Con respecto al sexo y la asociación con alteraciones en la secuencia de erupción, los resultados fueron del $54,8 \%$ para el femenino y el $45,2 \%$ para el masculino $(p=0,600)$. Conclusiones: Se encontró asociación significativamente estadística entre la enfermedad infecciosa y la alteración en la cronología con el retraso de la erupción de los dientes primarios. Se encontró asociación significativamente estadística entre la enfermedad infecciosa y las alteraciones en la secuencia de erupción; sin embargo, no fue tan categórica como la alteración en cronología, por lo que se sugiere profundizar la investigación.

Palabras clave: erupción dental, secuencia de erupción, gestante

\section{ABSTRACT}

Objective: To determine the association between the internal factors that influenced the pregnant woman up to the twentieth week of gestation, producing alterations in the eruption of the primary teeth of her children. SPECIFIC OBJECTIVES First; To determine the association of the factors that influenced the pregnant woman up to the twentieth week of gestation and the alterations in the chronology of eruption of the primary teeth of her children. Second; to determine the association of the factors that influenced the pregnant woman during the twentieth week of gestation and the alterations in the eruption sequence of the primary teeth of her children and third; to determine the association of the diseases produced in the gestation period and the repercussion in the alteration of the chronology and eruption sequences. Methodology: The present study will be observational, analytical retro prospective population and sample is 150 children, assisted in the service of growth and development (CRED). That they present some alteration in the chronology and / or sequence of eruption of the temporary teeth and the clinical histories of their respective mothers, who come to the Center of Maternal and Child Health Pachacutec Peru Korea Lima Callao, 2017, that fulfill the following CRITERIA OF INCLUSION Mothers that agree to participate with their youngest child in the research project, Children between 6 to 31 months of age who attend the service of growth and development (CRED), that presents some alteration in the chronology or sequence of eruption. Children with normal weight and height and with exclusive breastfeeding for the first six months of life. Mothers who have their complete medical records with their pregnancy controls, who belong to the health center between 20 to 35 years. EXCLUSION CRITERIA, Mothers or children that do not belong to the jurisdiction 
Alteraciones en la cronología y secuencia de erupción de dientes primarios asociadas a factores que influyeron hasta la veinteava semana de gestación de pacientes que acuden al Centro de Salud

of the health center. Children with mal nutrition or with a diet based on formula (artificial). Children with some syndrome or premature. Children with systemic diseases. Results: The factors that influenced the gestation that are found and that are evaluated in the middle of the statistical test in the square and the tests are worked at a level of significance of 0.05 . The association between infectious diseases and alterations in chronology has been found in diseases such as the ITU which is the most common $82.8 \%$ with the level of significance $\left(\mathrm{P}^{\circ}\right)$ $p=0.000$ ) The association between infectious diseases and alterations In the sequence we have found that diseases such as UTI in $88.1 \%$ with a level of significance $\left.\left(P^{\circ}\right) p=0.008\right)$ Finding lower percentages in diseases such as hypertension with $4.1 \%$, mental health $3.3 \%$ nutritional diseases $9.8 \%$ in what is chronology. In the sequence was found for hypertension $2.4 \%$ for mental health $2.4 \%$ nutritional diseases $7.1 \%$. Respect to the sex with association in alterations in the chronology of the feminine eruption $49.2 \%$ and masculine $50.8 \%, \mathrm{p}^{\circ}=0.271$. Respect to the sex with association in alterations in the sequence of feminine eruption $54.8 \%$ and masculine $45.2 \%$, $\mathrm{p}^{\circ}=0.600$. Conclusions: $A$ relationship was found between the statistics and the alteration in the chronology with the delay of the eruption of the primary teeth. A relationship between the statistic, the infectious one and the alterations in the sequence of the eruption have been found, however, it is not as categorical as the alteration in the chronology.

Keywords: dental eruption, eruption sequence, pregnant

\section{INTRODUCCIÓN}

La erupción de los dientes primarios es uno de los primeros signos que nos indica el crecimiento y la maduración del niño. Estas cronología y secuencia de erupción fueron estudiadas por Kronfeld y Logan, y posteriormente fueron modificadas por McCall y Schour. Ellos elaboraron una tabla de la cronología y secuencia determinada para la erupción de los dientes primarios. Es bastante conocido que el niño que está bajo de peso o desnutrido tiene una alteración en la cronología de erupción que se refleja en un retraso. Ha sido ampliamente estudiado que la lactancia exclusiva durante los seis primeros meses de vida ayuda a una correcta erupción de los dientes primarios; sin embargo, es frecuente ver en niños que han recibido lactancia exclusiva y están en su peso correcto presentar dicha alteración en la cronología o secuencia de erupción. Esto podría explicarse por factores que influyeron los primeros meses de vida intrauterina $\left({ }^{1}\right)$.

En el periodo de gestación se origina la formación de los dientes primarios, entre las cuatro y seis semanas de vida intrauterina, cuando se forma la corona con la banda epitelial primaria, seguida por la lámina dentaria, y termina a partir del quinto mes, en el que empieza a formarse la raíz. Existen otros factores que influyen en el periodo de gestación como la anemia, la desnutrición, el estrés, la depresión, enfermedades de diversa índole y factores ambientales, socioeconómicos, culturales y religiosos $\left(^{2}\right)$.

La presente investigación busca encontrar asociaciones en los distintos factores que influyeron en el periodo de embarazo y que pueden repercutir en la alteración de la cronología o la secuencia de erupción de los dientes primarios. Una vez identificada esta relación, lo que se buscará es crear estrategias para abordar esta problemática en forma multidisciplinaria con todas las áreas que intervienen durante el periodo de gestación: medicina, psicología, nutrición, obstetricia, enfermería y odontología. 
La cronología y secuencia de erupción se inicia con la aparición del diente primario en la cavidad bucal. El momento en el que rompe la mucosa y emerge se conoce como erupción dental y es un proceso que comprende varias fases, desde lo embriológico hasta diversos movimientos de desplazamiento dentro de la arcada $(1,2)$.

La erupción de los dientes primarios posee una gran variabilidad, con factores que la afectan como la raza, el clima, la nutrición, entre otras. Además, es útil tener siempre presente el patrón de erupción promedio para determinar si hay adelantos o retrasos notorios en la dentición primaria $(3,4)$. Se encuentra, según los estudios, mayor variabilidad en la cronología de erupción de los dientes primarios que en su secuencia $\left(^{5}\right)$.

En el periodo de gestación, la formación de los dientes primarios empieza entre las cuatro y seis semanas de vida intrauterina $(\mathrm{VI})$, periodo en el cual se forma la corona con la banda epitelial primaria, seguida por la lámina dentaria. De la séptima a la octava semana, aparecen los brotes o yemas. Entre la novena y la decimotercera semanas, se da el periodo de casquete o caperuza. Entre la decimocuarta y la decimoctava semanas se produce el periodo de campana. A partir del quinto mes, empieza a formarse la raíz, que en la literatura se suele llamar comienzo de la erupción, por la fuerza que da el crecimiento de la raíz. En las gestantes, los controles se realizan semanalmente; por ello, el presente trabajo abarca hasta la vigésima semana, en la que empieza la formación de la raíz ( $\left.{ }^{6}\right)$.

El proceso eruptivo se inicia con la calcificación de la raíz, una vez terminada la calcificación de la corona. Según estudios previos, este proceso se rige por un control endocrino que es el resultado de la acción simultánea de diversos fenómenos. Este se produce aproximadamente hasta la vigésima semana de VI $(7,8)$.

Existen diferentes estudios que mencionan una serie de factores que pueden influenciar en la cronología y secuencia de erupción. Asimismo, se establecen asociaciones entre el desarrollo dentario, el crecimiento general del cuerpo y la maduración del esqueleto durante el periodo de gestación (9-11).

\section{MATERIALES Y MÉTODOS}

La presente investigación uso el método de observación estructurada. Se pidió autorización a la Dirección de Salud del Gobierno Regional del Callao para la realización del estudio en el Centro de Salud Materno Infantil Pachacútec Perú-Corea, perteneciente a la Microrred Pachacútec de la Red de Salud de Ventanilla, parte de la Dirección Regional de Salud del Callao (Diresa-Callao). El estudio se llevó a cabo en el servicio de crecimiento y desarrollo (CRED) que pertenece al servicio de enfermería, al cual acuden los niños para sus vacunas y es por ello que van desde los 2 hasta los 18 meses de edad. Esto permitió tener una población identificable para los fines del estudio. Se solicitó, además, el permiso a las madres para realizar el examen a sus hijos mediante un consentimiento informado.

Para identificar a los niños que presentaban alteraciones en la cronología o secuencia de erupción, estos debían cumplir los criterios de selección (inclusión y exclusión) anteriormente mencionados. Se evaluó a los niños según la tabla cronológica del desarrollo de la dentición humana de Logan y Kronfeld, que fue modificada posteriormente por McCall y Schour, usando los valores iniciales de erupción de cada diente primario como referente de una correcta 
Alteraciones en la cronología y secuencia de erupción de dientes primarios asociadas a factores que influyeron hasta la veinteava semana de gestación de pacientes que acuden al Centro de Salud Materno Infantil Pachacútec Perú-Corea (Callao, 2017)

cronología y secuencia. Se tomó el valor máximo indicado en la tabla para hablar de retraso en la erupción, con la indicación de que el diente no debe haber cortado la encía para considerarlo con retardo de erupción. Se usó una ficha de recolección de datos para indicar los meses y el tipo de alteración.

Se realizó un examen clínico de la cavidad oral de niños de diferentes edades y se comprobó la alteración en la cronología o la secuencia. Se pidió a la madre su número de historia clínica para poder, mediante la ficha de recolección de datos, identificar los factores que influyeron en su gestación. Se seleccionó a las madres que tuvieran sus historias clínicas completas, con su evaluación médica obstétrica, nutricional, psicológica y odontológica, y sus exámenes de diagnósticos completos. Las fichas de recolección de datos fueron validadas por cuatros expertos en el área.

Los factores que influyen durante la gestación se han dividido en cuatro grupos. El primero son las enfermedades infecciosas, que agrupan a enfermedades bacterianas, micóticas, virales y parasitarias. El segundo grupo de enfermedades son las hipertensivas, que se producen cuando la gestante sufre de alteraciones en la presión arterial durante el periodo de gestación. En la actualidad, un grupo de mucha importancia es el de las enfermedades mentales, que agrupa la depresión y el estrés durante el embarazo. Por último, está el grupo de enfermedades carenciales, que incluye problemas nutricionales, anemia, etc. Cabe indicar que una gestante controlada es aquella que ha sido evaluada por todos los servicios, lo que permite tener una mirada retrospectiva y determinar con mayor precisión los factores que pueden alterar la cronología o la secuencia de erupción.

Este presente estudio es de tipo trasversal, observacional, estructural y prospectivo por la evaluación del niño, y retrospectivo con referencia a la madre.

\section{CRITERIOS DE INCLUSIÓN}

- Madres que acepten participar con su hijo en el proyecto de investigación.

- Niños entre 6 y 31 meses que acuden al servicio de crecimiento y desarrollo (CRED) del Centro de Salud Materno Infantil Pachacútec Perú-Corea, y que presentan alguna alteración en la cronología o secuencia de erupción dentaria.

- Niños con peso y talla normal.

- Niños con lactancia exclusiva los primeros seis meses de vida.

- Madres que tengan sus historias clínicas completas con sus controles de gestación, que pertenezcan al centro de salud.

- Gestantes entre 20 y 35 años.

\section{CRITERIOS DE EXCLUSIÓN}

- Madres o niños que no pertenezcan a la jurisdicción del centro de salud.

- Niños con desnutrición.

- Niños con alimentación a base de fórmula (artificial).

- Niños con algún síndrome o enfermedades sistémicas.

- Niños prematuros.

\section{ANÁLISIS ESTADÍSTICO}

El procesamiento y análisis estadístico de los datos se realizó por medio del programa estadístico SPSS versión 22. Los factores que influyeron en la gestación se evaluaron por medio de la prueba estadística Chi cuadrado. Las pruebas se trabajaron a un nivel de significancia de 0,05 . 


\section{RESULTADOS}

Los factores que influyeron en la gestación que se encontraran se evaluaron por medio de la prueba estadística chi cuadrado y las pruebas se trabajarán a un nivel de significancia de 0,05.
La asociación entre las enfermedades infecciosas y las alteraciones en la cronología se halló en males como las infecciones del tracto urinario (ITU), que son las más comunes $(82,8 \%)$, con un nivel de significancia $p=0,000$ (tabla 1 ).

Tabla 1. Asociación ENTRE ENFERMEdAdes InfECCIOSAS Y ALTERACIONES EN LA CRONOLOGÍA DE ERUPCIÓN

\begin{tabular}{|l|c|c|c|c|c|c|c|c|c|c|}
\hline Alteraciones & $\begin{array}{c}\text { Enf. } \\
\text { infecciosas }\end{array}$ & $\%$ & $\begin{array}{c}\text { Enf. } \\
\text { hiperten- } \\
\text { sivas }\end{array}$ & $\%$ & $\begin{array}{c}\text { Enf. } \\
\text { salud } \\
\text { mental }\end{array}$ & $\%$ & $\begin{array}{c}\text { Enf. } \\
\text { nutri- } \\
\text { cionales }\end{array}$ & $\%$ & No & Total \\
presentó & & & \\
\hline $\begin{array}{l}\text { Presenta } \\
\text { No presenta }\end{array}$ & 101 & 82,8 & 5 & 4,8 & 4 & 3,3 & 12 & 9,8 & 0 & 122 \\
\hline
\end{tabular}

Chi cuadrado de Pearson: $p^{\star}$ estadísticamente significativo $(p=0,00)$

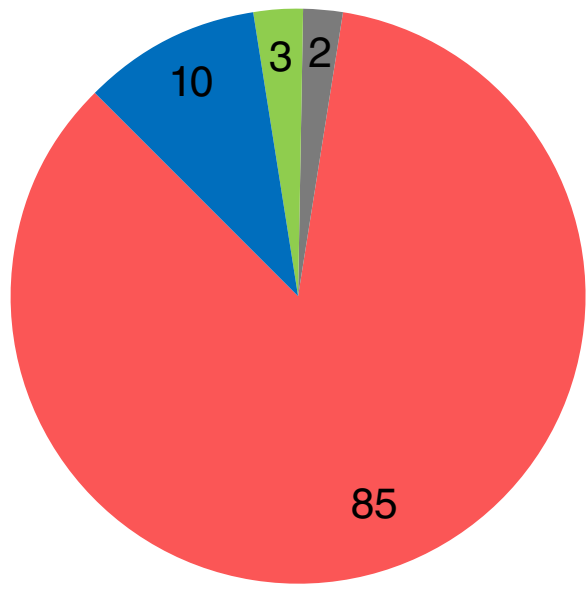

INFECCIÓN DEL TRACTO URINARIO (ITU)

ESTREPTOCOCO GRUPO B
VAGINOSIS

ITS SÍFILIS

GráFICO 1. EnFERMEDADES INFECCIOSAS DURANTE EL EMBARAZO

La asociación entre las enfermedades infecciosas y las alteraciones en la secuencia se mostró en enfermedades como las ITU $(88,1 \%)$, con un nivel de significancia $p=0,008$ (tabla 2)

Se hallaron porcentajes menores en enfermedades como la hipertensión $(4,1 \%)$, la salud mental $(3,3 \%)$, las enfermedades nutricionales $(9,8 \%)$, con respecto a la cronología.
En el caso de la secuencia, se encontró para hipertensión un 2,4\%; para salud mental, un $2,4 \%$, y para enfermedades nutricionales, un $7,1 \%$.

Con relación al sexo, en el caso de las alteraciones en la cronología de erupción, fue del $49,2 \%$ para el femenino y el $50,8 \%$ para el masculino, con $p=0,271$ (tabla 3); mientras que en el caso de las alteraciones en la secuencia de erupción fue del $54,8 \%$ para el femenino y del 
Alteraciones en la cronología y secuencia de erupción de dientes primarios asociadas a factores que influyeron hasta la veinteava semana de gestación de pacientes que acuden al Centro de Salud Materno Infantil Pachacútec Perú-Corea (Callao, 2017)

TABLA 2. AsOCIACIÓN ENTRE ENFERMEDADES INFECCIOSAS Y ALTERACIONES EN LA SECUENCIA DE ERUPCIÓN

\begin{tabular}{|l|c|c|c|c|c|c|c|c|c|c|}
\hline Alteraciones & $\begin{array}{c}\text { Enf. } \\
\text { infec- } \\
\text { ciosas }\end{array}$ & $\begin{array}{c}\text { Enf. } \\
\text { hiperten- } \\
\text { sivas }\end{array}$ & $\begin{array}{c}\text { Enf. } \\
\text { salud } \\
\text { mental }\end{array}$ & $\begin{array}{c}\text { Enf. } \\
\text { nutri- } \\
\text { cionales }\end{array}$ & $\begin{array}{c}\text { No } \\
\text { presentó }\end{array}$ & Total \\
\hline PRESENTA & 37 & 88,1 & 1 & 2,4 & 1 & 2,4 & 3 & 7,1 & 0 & 42 \\
\hline NO PRESENTA & 65 & 60,2 & 4 & 3,7 & 3 & 2,8 & 10 & 9,3 & 26 & 108 \\
\hline
\end{tabular}

Chi cuadrado de Pearson: $p^{*}$ estadísticamente significativo $(p=0,008)$

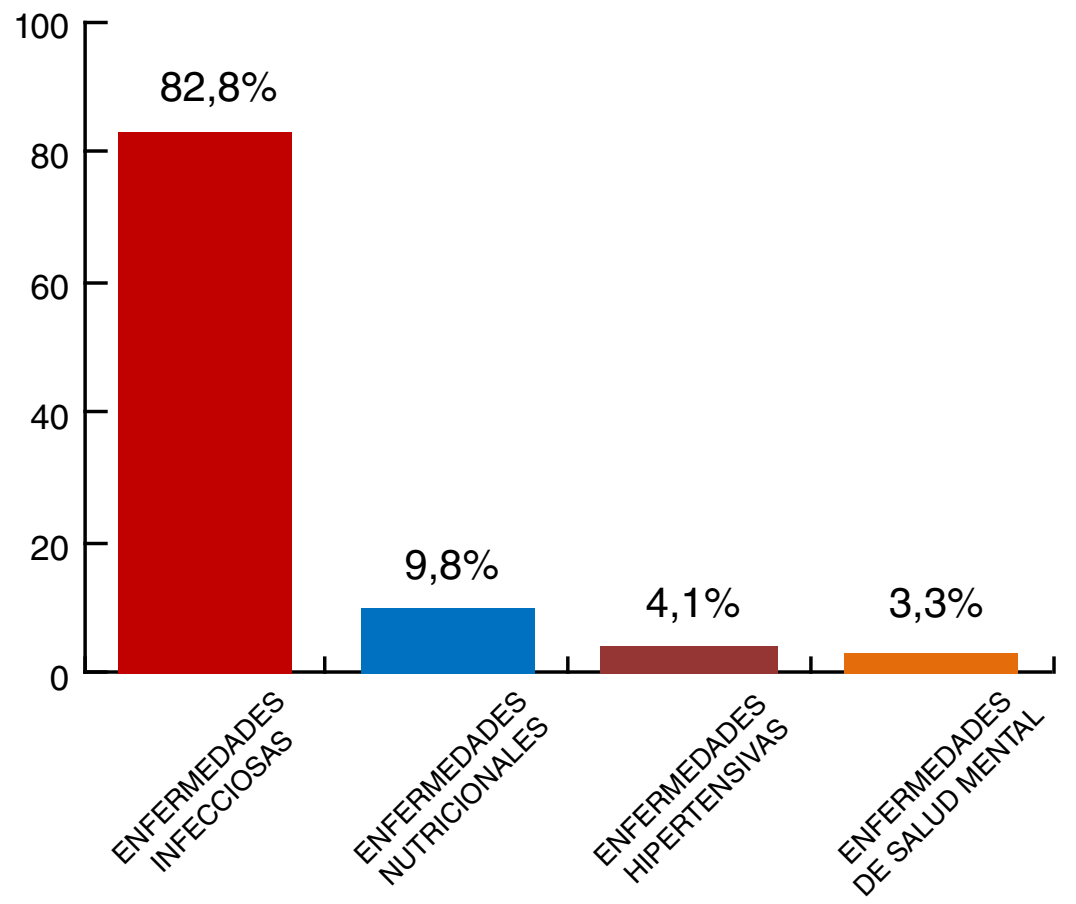

Gráfico 2. Factores asociados a gestantes

TABLA 3. Asociación ENTRE alteraciones EN LA CRONOLOGÍA de ERUPCIÓN CON EL SEXo

\begin{tabular}{|l|c|c|c|c|c|c|}
\hline Alteraciones & Femenino & $\%$ & Masculino & $\%$ & Total & Porcentaje total \\
\hline Presenta & 60 & 49,2 & 62 & 50,8 & 122 & $100 \%$ \\
\hline No presenta & 17 & 60,7 & 11 & 39,3 & 28 & $100 \%$ \\
\hline
\end{tabular}

Chi cuadrado de Pearson: $p^{*}(p=0,271)$ 


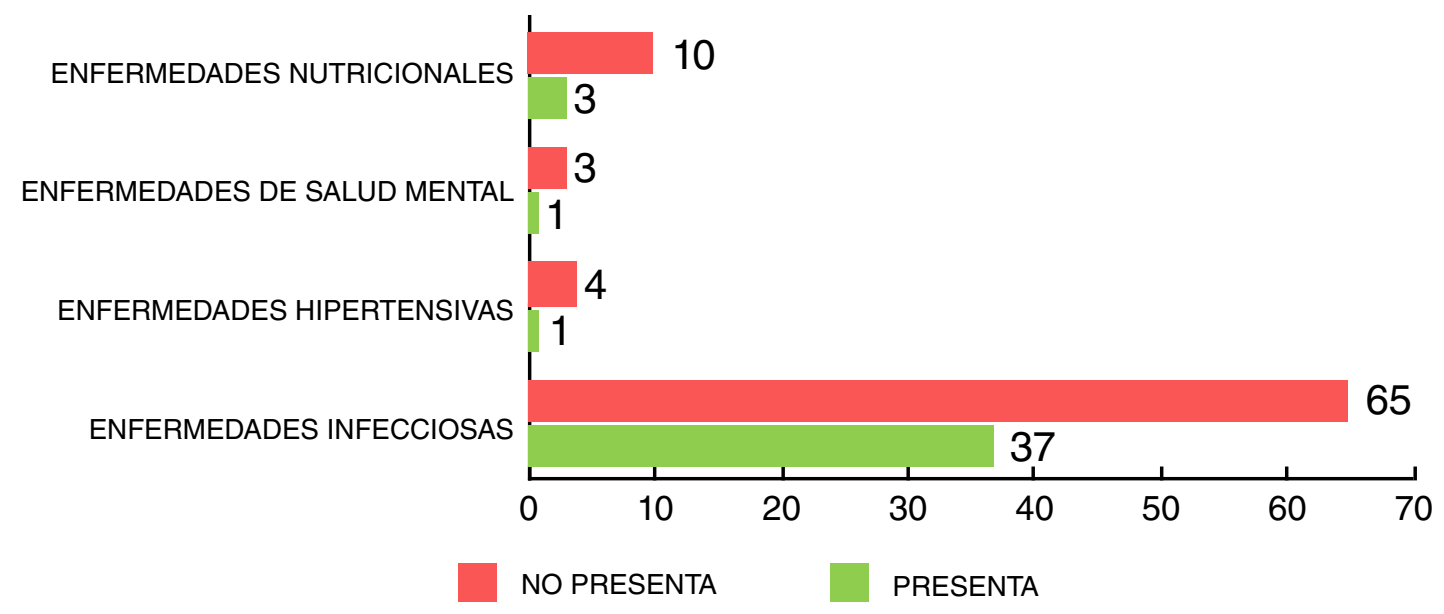

Gráfico 3. Alteraciones en La SECUENCIA de ERUPCión

TABla 4. Asociación ENTRE LAS ALTERACIONES EN LA SECUENCIA DE ERUPCIÓN Y EL SEXo

\begin{tabular}{|l|c|c|c|c|c|}
\hline Alteraciones & Femenino & $\%$ & Masculino & $\%$ & Total \\
\hline Presenta & 23 & 54,8 & 19 & 45,2 & 42 \\
\hline No presenta & 54 & 50,0 & 54 & 50,0 & 108 \\
\hline
\end{tabular}

Chi cuadrado de Pearson: $p^{*}(p=0,600)$

$45,2 \%$ para el masculino, con $p=0,600$ (tabla 4).

\section{DISCUSIÓN}

Actualmente, existen pocos estudios similares. Algunos de ellos coinciden en que una serie de factores pueden influir en la cronología y la secuencia de erupción de los dientes primarios. Asimismo, se refieren unas series de asociaciones positivas entre el desarrollo dentario, el crecimiento general del cuerpo y la maduración del esqueleto en el periodo de gestación (9-11).

La investigación tendrá importancia teórica debido a que, de probarse esta asociación, se contribuirá de manera confiable a los conocimientos científicos en esta área, ya que se determinarán los factores que influyeron durante la gestación y permitirá identificar las enfermedades durante el periodo de gestación que podrían alterar la cronología y secuencia de erupción de los dientes primarios. Además, tendrá importancia social porque las gestantes podrán disminuir el riesgo de exponerse a estos factores y controlar mejor su periodo de gestación.

Gupta y Magnusson (21, 22) encontraron una erupción un poco más adelantada en el caso del sexo femenino, aunque solo este último obtuvo resultados estadísticamente significativos en el caso de los incisivos centrales primarios inferiores y los segundos molares.

Folayan et al. $\left({ }^{9}\right)$ encontraron retraso en la erupción en los niños de madres que estaban medicamente comprometidos.

En nuestro estudio, hallamos gestantes que habían tomado fármacos. Por ejemplo, para las ITU tomaron cefazolina 
Alteraciones en la cronología y secuencia de erupción de dientes primarios asociadas a factores que influyeron hasta la veinteava semana de gestación de pacientes que acuden al Centro de Salud

y nitrofurantoina, para la sífilis tomaron penicilina $G$ benzatinica, para la vaginosis tomaron metronidazol a partir de la semana 14 , porque atraviesa la barrera placentaria. En nuestra investigación, la patología más encontrada fueron las infecciones del tracto urinario, las cuales se dieron en el primer trimestre del embarazo y obligaron a las madres a tomar antibioticoterapia de elección como las cefalosporinas, lo que pudo alterar de alguna manera la formación de los dientes primarios. Se necesita más investigación del área para determinar si son medicamentos teratógenos.

Ramírez et al. $\left({ }^{10}\right)$ analizaron el tamaño muestral de este tipo de estudios cronológicos, para lo cual emplearon muestras parecidas a la nuestra, aunque con un método longitudinal.

Psoter et al. ( $\left.{ }^{7}\right)$ trabajaron muestras mayores que les dieron resultados más significativos. De la misma manera, la edad de aparición del primer molar superior derecho se alejó bastante de los rangos aceptados en erupción de dientes primarios y de los valores obtenidos por otros autores. Los resultados del presente trabajo arrojaron retraso en la erupción de la segunda molar inferior.

Los estudios revisados muestran la influencia de determinados factores sobre la erupción, como la raza, que resulta determinante en las diferencias entre las distintas poblaciones, y también el sexo. Al estudiar las diferencias en la erupción de la dentición primaria de diversos países, encontramos que, según las investigaciones realizadas, los niños coreanos tienen una erupción más adelantada, seguidos por los niños españoles, que también presentan edades medias de emergencia tempranas, siendo Arabia Saudita la que presenta mayor retraso en la erupción de estos dientes ${ }^{(19-23)}$.
Existen autores que señalan varias causas posibles del retraso en la erupción dental, tal es el caso de Biondy, quien en 2008 señaló que, entre los factores involucrados en este proceso, se encuentran la edad, el sexo y la raza ${ }^{(24)}$. Sin embargo, el presente estudio se enfocó en una población rural de extrema pobreza que son migrantes de diferentes partes del Perú, por lo que se podría decir que nuestra muestra representaría una muestra real de las diversas poblaciones de nuestro país. En nuestra investigación, se encontró asociación significativa de las ITU con la alteración en cronología bien marcada y de menor forma en el caso de las alteraciones en secuencias; asimismo, el sexo no influyó en los resultados. Las enfermedades hipertensivas mentales y nutricionales se presentaron en menor cantidad, sin mayor significancia. No se encuentra mucha bibliografía con respecto a asociar factores de riesgos en gestación y alteraciones, por lo que se recomienda investigar dichas asociaciones con mayor profundidad.

\section{CONCLUSIONES}

1. Se encontró asociación significativamente estadística entre la enfermedad infecciosa y la alteración en la cronología con el retraso de la erupción de los dientes primarios.

2. Se encontró asociación significativamente estadística entre la enfermedad infecciosa y las alteraciones en la secuencia de erupción; sin embargo, no es tan categórica como la alteración en cronología. Se sugiere mayor análisis e investigación.

3. Respecto de las alteraciones en cronología y secuencia asociadas al sexo, no se encontró asociación.

4. La enfermedad que se presentó con mayor cantidad fueron las infecciones del tracto urinario (ITU) en los tres primeros meses (gráfico 1). 


\section{RECOMENDACIONES}

- Los resultados de la investigación nos indican que las enfermedades infecciosas tienen una asociación con las alteraciones de erupción de los dientes primarios; por ello, se recomienda mayor estudio sobre los fármacos que son ingeridos durante el periodo del embarazo.

- Se realiza este estudio con el fin de reorientar la formulación de estrategias preventivas en las áreas de medicina, enfermería, obstetricia y odontología para evitar o disminuir dichas alteraciones.
Contribución del autor: Luciano Álvaro Ordóñez y Denisse Pilar Carmen Aguilar Gálvez han participado en la concepción del artículo, la recolección de información, su redacción y la aprobación de la versión final.

Fuente de financiamiento: Autofinanciado.

Conflicto de intereses: LoS autores declaran no tener conflicto de interés de ningún tipo. 
Alteraciones en la cronología y secuencia de erupción de dientes primarios asociadas a factores

\section{REFERENCIAS BIBLIOGRÁFICAS}

1. Barbería Leache E. Erupción dentaria. Prevención y tratamiento de sus alteraciones. Pediatr Integral. 2001; 6 (3): 229-40.

2. Choi NK, Yang KH. A study on the eruption timing of primary teeth in Korean children. ASDC J Dent Child. 2001; 68 (4): 244-9.

3. Psoter WJ, Morse DE, Pendrys DG, Zhang H, Mayne ST. Median ages of eruption of the primary teeth in white and Hispanic children from Arizona. Pediatr Dent. 2003; 25 (3): 257-61.

4. Holman D, Jones R. Longitudinal analysis of deciduous tooth emergence: II. Parametric survival analysis in Bangladeshi, Guatemalan, Japanese, and Javanese children. Am J Phys Anthropol. 1998; 105 (2): 209-30.

5. Moron B, Santana Y, Pirona M, Rivera L, Rincón M, Pirela A. Cronología y secuencia de erupción de dientes permanentes en escolares wayuu. Parroquia Idelfonso Vázquez. Municipio Maracaibo-Estado de Zulia. Acta Odontol Venez. 2006; 44 (1).

6. Liversidge HM, Molleson T. Variation in crown and root formation and eruption of human deciduous teeth. Am J Phys Anthropol. 2004; 123 (2): 172-80.

7. Bockmann MR, Hughes TE, Townsend GC. Genetic modeling of primary tooth emergence: a study of Australian twins. Twin Res Hum Genet. 2010; 13 (6): 573-81.

8. Folayan M, Owotade F. The timing of eruption of the primary dentition in Nigerian children. Am J Phys Anthropol. 2007; 134: 443-8.

9. Sahin F. Camurdan A. Factors affecting the timing of teething in healthy turkish infants; a prospective cohort study. Int J Paediatr Dent. 2008, 18 (4): 262-6.

10. Haddad AE, Correa MS. The relationship between the number of erupted primary teeth and the child's height and weight: a cross-sectional study. J Clin Pediatr Dent. 2005; 29 (4): 357-62.

11. Bello LL, Al-Jasser NM. Time of eruption of primary dentition in Saudi children. J Contemp Dent Pract. 2003; 4 (3): 65-75.

12. Catalá Pizarro M, Canut Brusola JA, Plasencia Alcina E. Evaluación crítica de los trabajos sobre cronología de erupción de la dentición temporal. Arch Odontoestomatol. 1986; 2 (6): 321-8.

13. Lunt RC, Law D. A review of the chronology of eruption of deciduous teeth. JADA. 1974; 89 (4): 872-9.

14. Hägg U, Taranger J. Dental development, dental age and tooth counts. A longitudinal study of the timing of tooth emergence in Swedish children from birth to 18 years. The Angle Orthod. 1985; 55 (2): 93-107.

15. Romero Maroto M, Sáez Gómez JM. Eruption of primary dentition--a grave health problem according to Spanish doctors of the XVI-XVIII centuries. J Den Res. 2009; 88 (9): 777-80. 
16. Leung AK. Teething. Am Fam Physician. 1989; 39 (2): 131-4.

17. Peretz B, Ram D, Hermida L, Otero MM. Systemic manifestations during eruption of primary teeth in infants. J Dent Child. 2003; 70 (2): 170-3.

18. Galili G, Rosenzweig KA, Klein H. Eruption of primary teeth and general pathologic conditions. ASDC J Dent Child. 1969; 36 (1): 51-4.

19. Logan, WHG, Kronfeld R. Development of human jaws and surrounding structures from birth to the age of fifteen years. J Am Dent Assoc. 1933; 20: 379-427.

20. Lunt RC, Law D. A review of the chronology of eruption of deciduous teeth. JADA. 1974; 89 (4): 872-9.

21. Kiran K, Swati T, Kamala BK, Jaiswal D. Prevalence of systemic and local disturbances in infants during primary teeth eruption: a clinical study. Eur $\mathrm{J}$ Paediatr Dent. 2011; 12 (4): 249-52.

22. Boj JR, Catalá M, García Ballesta C, Mendoza A, Planells P. Odontopediatría. La evolución del niño al adulto joven. Madrid: Ripano; 2010.

23. Nakata M, Wei S. Desarrollo del arco dental y oclusión. En: Guía oclusal en odontopediatría. Atlas a color. Japón: Amolca; 1989.

24. Torres CM. Desarrollo de la dentición. La dentición primaria. Rev Latinoam Ortod Odontopediat. 2012; 234 (2): 172-9.

25. Barbería E. Erupción dentaria. Prevención y tratamiento de sus alteraciones. Pediatr Integral. 2001; 6(3): 229-40.

26. Psoter WJ, Morse DE, Pendrys DG, Zhang H, Mayne ST. Median ages of eruption of the primary teeth in white and Hispanic children from Arizona. Pediatr Dent. 2003; 25 (3): 257-61.

27. Koch G, Modeér T, Poulsen S, Rasmussen P. Desarrollo dentario y oclusal normal. En: Odontopediatría. Enfoque clinico. Buenos Aires: Editorial Médica Panamericana; 1995.

28. Berkovitz BKB, Holland GR, Moxham BJ. Atlas en color y texto de anatomía oral, histología y embriología. 2.- ed. Madrid: Mosby; 1995.

29. Barbería E. Atlas de odontología infantil para pediatras y odontólogos. Madrid: Ripano; 2005.

30. Solano Reina E, Martín de Agar Valverde MC, Mendoza Mendoza A. Fisiología de la erupción dentaria. Av Odontoestomatol. 1987; 3 (2): 87-94. 